Bull. Korean Math. Soc. 41 (2004), No. 3, pp. 419426

\title{
RELATIVE PROJECTIVITY AND RELATED RESULTS
}

\author{
H. ANSARI-TOROGHY
}

\begin{abstract}
Let $R$ be a commutative Noetherian ring and let $M$ be an Artinian $R$-module. Let $M^{\prime \prime} \subseteq M^{\prime}$ be submodules of $M$. Suppose $F$ is an $R$-module which is projective relative to $M$. Then it is shown that

$$
\operatorname{Att}_{R}\left(H o m_{A}\left(F, M^{\prime}\right):_{H \circ m_{A}(F, M)} I^{n}\right), n \in N
$$

and

$$
\begin{aligned}
& \operatorname{Att}_{R}\left(\operatorname{Hom}_{A}\left(F, M^{\prime}\right):_{\operatorname{Hom}_{A}(F, M)} I^{n}\right. \\
& \left./ \operatorname{Hom}_{A}\left(F, M^{\prime \prime}\right):_{H_{0 m}(F, M)} I^{n}\right), n \in N
\end{aligned}
$$

are ultimately constant.
\end{abstract}

\section{Introduction}

Throughout of this paper $R$ will denote a commutative ring (with a nonzero identity).

Let $M$ be an $R$-module. A prime ideal $P$ of $R$ is said to be an associated prime of $M$ if there exists an element $x \in M$ such that $P=$ $(0: R R x)$ (see [2, Chapter 4]). The set of associated primes of $M$ is denoted by $A s s_{R}(M)$.

We shall follow Macdonald's terminology (see [4]) concerning secondary representation. So whenever an $R$-module $L$ has a secondary representation, then the set of attached primes of $L$ is denoted by $A t t_{R}(L)$.

Let $I$ be an ideal of $R$ and suppose that $F$ and $M$ are respectively a flat and an Artinian $R$-modules. Let $M^{\prime \prime} \subseteq M^{\prime}$ be submodules of $M$ and let $T=H_{0} m_{R}(F,-)$. In [3] it has shown that the sequences of sets $A t t_{R}\left(T\left(M^{\prime}\right):_{T(M)} I^{n}\right)$ and $A t t_{R}\left(T\left(M^{\prime}\right):_{T(M)} I^{n} / T\left(M^{\prime \prime}\right):_{T(M)} I^{n}\right)$, $n \in N$, are ultimately constant.

Let $I, M, M^{\prime}$, and $M^{\prime \prime}$ be as in the above paragraph and let $F$ be an $R$-module (not necessarily flat). In this paper we will generalize the above mentioned results in the case that $F$ is projective relative to $M$.

Received December 6, 2002.

2000 Mathematics Subject Classification: 13E05.

Key words and phrases: associated primes, relative projectivity, attached primes. 
We recall that $F$ is projective relative to $M$ (or $F$ is M-projective) if and only if for any homomorphic image $N$ of $M$, the homomorphism $\operatorname{Hom}_{R}(F, M) \rightarrow \mathrm{Hom}_{R}(F, N)$ is epic (see [1]).

\section{Auxiliary results}

REMark 2.1. (a) If $F$ is an $R$-module which is $M$-projective and $X$ a submodule or a homomorphic image of $M$, then $F$ is $X$-projective.

(b) If $\left(M_{i}\right)_{i \in I}$ is a finite family of $R$-modules and $F$ is projective relative to $M_{i}$ for each $i \in I$, then $F$ is projective relative to $\oplus_{i \in I} M_{i}$.

(c) If $F$ is a $M$-projective and

$$
0 \rightarrow K \rightarrow M \rightarrow L \rightarrow 0
$$

is an exact sequence of $R$-modules and $R$-homomorphisms with middle term $M$, then

$$
0 \rightarrow \operatorname{Hom}_{R}(F, K) \rightarrow \operatorname{Hom}_{R}(F, M) \rightarrow \operatorname{Hom}_{R}(F, L) \rightarrow 0
$$

is also an exact sequence.

Lemma 2.2. Let $R$ be a commutative Noetherian ring and let $M$ be an Artinian $R$-module. Suppose $F$ is an $R$-module which is projective relative to $M$ and Let $E=\oplus P \in \Omega E(R / P)$, where $\Omega$ is a finite subset of $\operatorname{Max}(R)$ with $\operatorname{Ass}_{R}(M) \subseteq \Omega$. Set $T(-)=H_{o m_{R}}(-, E)$. Then $T(F)$ is injective relative to $T(M)$. (We recall that an $R$-module $X$ is injective relative to an $R$-module $H$ if and only if for any submodule $L$ of $H$, $\mathrm{Hom}_{R}(H, X) \rightarrow \mathrm{Hom}_{R}(L, X)$ is epic (see [1]).)

Proof. Let

$$
0 \rightarrow L \rightarrow T(M)
$$

be an exact sequence of $R$-modules and $R$-homomorphisms. Hence

$$
T(T(M)) \rightarrow T(L) \rightarrow 0
$$

is an exact sequence. But $T(T(M)) \cong M$ by [3, Lemma 3.1]. It follows that $F$ is projective relative to $T(T(M))$. Hence

$$
H_{R}\left(F, T(T(M)) \rightarrow H o m_{R}(F, T(L)) \rightarrow 0\right.
$$

is an exact sequence. It turns out that

$$
\mathrm{Hom}_{R}(T(M), T(F)) \rightarrow \mathrm{Hom}_{R}(L, T(F)) \rightarrow 0
$$

is an exact sequence. Hence $T(F)$ is injective relative to $T(M)$ and the proof is complete. 
Lemma 2.3. Let $M_{1}$ and $M_{2}$ are $R$-modules. Suppose $F$ is an $R$ module which is projective relative to both $M_{1}$ and $M_{2}$. Then

$$
H_{o m}\left(F, M_{1}+M_{2}\right)=H o m_{R}\left(F, M_{1}\right)+H o m_{R}\left(F, M_{2}\right) \text {. }
$$

Proof. Put $T=\operatorname{Hom}_{R}(F,-)$. It is clear that

$$
T\left(M_{1}\right)+T\left(M_{2}\right) \subseteq T\left(M_{1}+M_{2}\right) .
$$

To see the reverse inclusion let $f \in T\left(M_{1}+M_{2}\right)$. Since $F$ is projective relative to $M_{1} \oplus M_{2}$ by 2.1 , as in [3] (1.5), we can find $f_{i} \in T\left(M_{i}\right)$, $i=1,2$, such that $f=f_{1}+f_{2}$. So the proof is complete.

Lemma 2.4. Let $I$ be a finitely generated ideal of $R$ and let $M$ be an $R$-module. Suppose $F$ is an $R$-module which is projective relative to $M$. Put $T=H_{\text {om }}(F,-)$. Then $T(I M)=I T(M)$.

Proof. Let $x \in R$. Since $F$ is projective relative to $M$, from the exact sequence

$$
M \stackrel{x}{\rightarrow} x M \rightarrow 0
$$

we get the exact sequence

$$
T(M) \stackrel{x}{\rightarrow} T(x M) \rightarrow 0 .
$$

Hence $T(x M)=x T(M)$. Now the result follows from this and Lemma 2.3 .

Lemma 2.5. Let $S$ be another commutative ring and let $\phi: R \rightarrow S$ be a ring homomorphism. Let $M$ be an $S$ module. Suppose $F$ is an $R$ module which is projective relative to $M$ when regarded as $R$-module. Then as $S$-modules, $F \otimes_{R} S$ is projective relative to $M$.

Proof. Let

$$
M \rightarrow N \rightarrow 0
$$

be an exact sequence of $S$-modules and $S$-homomorphisms. Hence, as $R$-modules and $R$-homomorphisms,

$$
\operatorname{Hom}_{R}(F, M) \rightarrow \operatorname{Hom}_{R}(F, N) \rightarrow 0,
$$

is an exact sequence. But as $S$-modules,

$$
\operatorname{Hom}_{S}\left(F \otimes_{R} S, M\right) \cong \operatorname{Hom}_{R}(F, M) .
$$

It follows that

$$
H o m_{S}\left(F \otimes_{R} S, M\right) \rightarrow H o m_{S}\left(F \otimes_{R} S, N\right) \rightarrow 0 .
$$

is an exact sequence. So the proof is complete. 


\section{Main results}

Throughout this section $A$ will denote a commutative Noetherian ring with a non-zero identity.

We recall the concept of coassociated primes from [9].

Definition. An $A$-module $L$ is cocyclic if $L \subseteq E(A / P)$ for some maximal ideal $P$ of $A$. Also a prime ideal $P$ of $A$ is said to be a coassociated prime of $M$ if there exists a cocyclic homomorphic image $L$ of $M$ such that $P=\left(0:{ }_{A} L\right)$. The set of coassociated primes of $M$ is denoted by $\operatorname{Coass}_{A}(M)$.

Lemma 3.1. Let $M$ be an Artinian $A$-module and suppose $F$ is an $A$-module which is projective relative to $M$. Then we have the following.

(i) $\operatorname{Hom}_{A}(F, M) \neq 0$ if only if there exists $P \in \operatorname{Att}_{A}(M)$ such that $P \subseteq Q$ for some $Q \in \operatorname{Coass}_{A}(F)$.

(ii) If $M$ is a $P$-secondary $A$-module and $\operatorname{Hom}_{A}(F, M) \neq 0$, then $H_{A}(F, M)$ is a $P$-secondary A-module.

Proof. (i) $(\Rightarrow)$ We can use the same technique used in [3] (3.2). To see the converse, let $P \in \operatorname{Att}_{A}(M)$ and let $P \subseteq Q$ for some $Q \in$ Coass $_{A}(F)$. Then there exist maximal ideals $P_{1}$ and $P_{2}$ such that $P \in$ $A s s_{A}\left(H_{0 m}\left(M, E\left(A / P_{1}\right)\right)\right)$ and $Q \in A s s_{A}\left(H_{A} m_{A}\left(M, E\left(A / P_{2}\right)\right)\right)$ by $[9$, 1.7]. Set $\Omega=A s s_{A}(M) \cup\left\{P_{1}, P_{2}\right\}$ and $D(-)=H o m_{A}\left(-, \oplus_{P \in \Omega} E(A / P)\right)$. It implies that $P \in A s s_{A}(D(M))$ and $Q \in A s s_{A}(D(F))$. Since $D(F)$ is injective relative to $D(M)$ by Lemma 2.2 , from the exact sequence

$$
0 \rightarrow A / P \rightarrow D(M)
$$

we obtain the exact sequence

$$
H o m_{A}(D(M), D(F)) \rightarrow H o m_{A}(A / P, D(F)) \rightarrow 0 .
$$

Further,

$$
0 \rightarrow A / Q \rightarrow D(F)
$$

is an exact sequence so that $\operatorname{Hom}_{A}(A / Q, D(F)) \neq 0$. Also $D(F)$ is injective relative to $A / P$ by Remark 2.1. Hence from the exact sequence

$$
A / P \rightarrow A / Q \rightarrow 0
$$

we get the exact sequence

$$
0 \rightarrow \operatorname{Hom}_{A}(A / Q, D(F)) \rightarrow \operatorname{Hom}_{A}(A / P, D(F)) .
$$

Hence we have $\operatorname{Hom}_{A}(A / P, D(F)) \neq 0$. This in turn implies that

$$
H o m_{A}(D(M), D(F)) \neq 0 \text {. }
$$


But by $[3,3.1], M \cong D(D(M))$. Hence we have

$$
\operatorname{Hom}_{A}(F, M) \cong \operatorname{Hom}_{A}(D(M), D(F)) \neq 0 .
$$

(ii) Let $r \in R$. Then $M \stackrel{r}{\rightarrow} M$ is nilpotent or surjective. Hence $\operatorname{Hom}_{R}(F, M) \stackrel{r}{\rightarrow} \operatorname{Hom}_{R}(F, M)$ is either nilpotent or surjective. Therefore, $\operatorname{Hom}_{R}(M, E)$ is a $P$-secondary module and the proof is complete.

Corollary 3.2. (See $[3,3.2]$.) Let $M$ be an Artinian $A$-module and suppose $F$ is a flat $A$-module. Then we have the following.

(i) $\operatorname{Hom}_{A}(F, M) \neq 0$ if only if there exists $P \in \operatorname{Att}_{A}(M)$ such that $P \subseteq Q$ for some $Q \in$ Coass $_{A}(F)$.

(ii) If $M$ is a $P$-secondary $A$-module and $\operatorname{Hom}_{A}(F, M) \neq 0$, then $H_{A} m_{A}(F, M)$ is a $P$-secondary $A$-module.

Proof. By [6, Theorem 5], T= $\operatorname{Hom}_{A}(F,-)$ is an exact functor over the category of Artinian $A$-modules. It implies that $F$ is projective relative to $M$. Hence the result follows from Lemma 3.1.

Theorem 3.3. Let $M$ be an Artinian A-module, and suppose that $F$ is an $A$-module which is projective relative to $M$. Then $\operatorname{Hom}_{A}(F, M)$ has a secondary representation and we have

$$
\begin{aligned}
& \operatorname{Att}_{A}\left(\operatorname{Hom}_{A}(F, M)\right) \\
= & \left\{P \in \operatorname{Att}_{A}(M): P \subseteq Q \quad \text { for some } Q \in \operatorname{Coass}_{A}(F)\right\} .
\end{aligned}
$$

Proof. Let $M=\sum_{i=1}^{r} M_{i}$ be a minimal secondary representation for $M$, where each $M_{i}$ is $P_{i}$-secondary. Suppose for $i=1,2, \ldots, t$, there exists $P_{i} \subseteq Q_{i}$ for some $Q_{i} \in$ Coass $_{A}(F)$, while this doesn't hold for $i=t+1, t+2, \ldots, r$. Let $T(-)=H_{o m}(F,-)$. Then by Lemma 3.1, for $i=1,2, \ldots, t, T\left(M_{i}\right) \neq 0$ and it is $P_{i}$-secondary. Hence by Lemma 2.3 , we have

$$
T(M)=H_{A}(F, M)=\sum_{i=1}^{t} T\left(M_{i}\right) .
$$

So $\operatorname{Hom}_{A}(F, M)$ has a secondary representation. We claim that the above decomposition is in fact a minimal one. To see this let for an integer $j$ with $1 \leq j \leq t$,

$$
T\left(M_{j}\right) \subseteq \sum_{\substack{i=1 \\ i \neq j}}^{t} T\left(M_{i}\right)
$$


It implies that

$$
T(M)=\operatorname{Hom}_{A}(F, M)=\sum_{\substack{i=1 \\ i \neq j}}^{r} T\left(M_{i}\right)=T\left(\sum_{\substack{i=1 \\ i \neq j}}^{r} M_{i}\right) .
$$

Now from

$$
0 \rightarrow \sum_{\substack{i=1 \\ i \neq j}}^{r} M_{i} \rightarrow M \rightarrow M / \sum_{\substack{i=1 \\ i \neq j}}^{r} M_{i} \rightarrow 0
$$

by using Lemma 2.1 , we obtain the exact sequence

$$
0 \rightarrow T\left(\sum_{\substack{i=1 \\ i \neq j}}^{r} M_{i}\right) \rightarrow T(M) \rightarrow T\left(M / \sum_{\substack{i=1 \\ i \neq j}}^{r} M_{i}\right) \rightarrow 0 .
$$

It implies that

$$
T\left(M / \sum_{\substack{i=1 \\ i \neq j}}^{r} M_{i}\right)=0
$$

But

$$
M / \sum_{\substack{i=1 \\ i \neq j}}^{r} M_{i} \cong M_{j} /\left(M_{j} \cap \sum_{\substack{i=1 \\ i \neq j}}^{r} M_{i}\right)
$$

is $P_{j}$-secondary. It turns out that

$$
\operatorname{Att}_{A}\left(M / \sum_{\substack{i=1 \\ i \neq j}}^{r} M_{i}\right)=\left\{P_{j}\right\}
$$

Hence by Lemma 3.1 , we have $T\left(\sum_{\substack{i=1 \\ i \neq j}}^{r} M_{i}\right) \neq 0$ which is a contradiction. This completes the proof.

REMARK 3.4. Theorem 3.3 extends [3, Theorems 3.3, 3.5, and 3.6] because as we mentioned in the proof of 3.2 , every flat $A$-module is projective relative to every Artinian $A$-module.

Corollary 3.5. Let $M$ be a finitely generated $A$-module and suppose $F$ is an $A$-module which is projective relative to $M$. Let $P$ be a maximal ideal of $A$ such that $P \in \operatorname{Coass}_{A}(F)$ and let $i \geq 0$. Then $H_{P}^{i}(M)$, and $\operatorname{Hom}_{A}\left(F, H_{P}^{i}(M)\right)$ have the same set of attached prime ideals, so that the following are equivalent.

(i) $H_{P}^{i}(M) \neq 0$

(ii) $\operatorname{Hom}_{A}\left(F, H_{P}^{i}(M)\right) \neq 0$. 
Proof. It is straightforward to see that $H_{P}^{i}(M)$ is an Artinian $A$ module. Further since $A s s_{A}\left(H_{P}^{i}(M)\right) \subseteq\{P\}$, every $P^{\prime} \in A t t_{A}\left(H_{P}^{i}(M)\right)$ is contained in $P$. Hence by using Theorem 3.3, we have

$$
\operatorname{Att}_{A}\left(\operatorname{Hom}_{A}\left(F, H_{P}^{i}(M)\right)\right)=\operatorname{Att}_{A}\left(H_{P}^{i}(M)\right) \text {. }
$$

The result follows from this and the proof is complete.

Let $M$ be an $R$-module, and let $M^{\prime}$ be a submodule of $M$. Then for an ideal $I$ of $R$ it follows that

$$
\operatorname{Hom}_{R}\left(L, M^{\prime}\right):_{H o m_{R}(L, M)} I=\operatorname{Hom}_{R}\left(L,\left(M^{\prime}:_{M} I\right)\right) .
$$

TheOREM 3.6. Let $I$ be an ideal of $R$ and let $M$ be an Artinian $R$-module. Suppose $F$ is an $R$-module which is projective relative to $M$. Let $M^{\prime \prime} \subseteq M^{\prime}$ be submodules of $M$. Further let $T(-)=\operatorname{Hom}_{A}(F,-)$. Then the sequences of sets

$$
A t t_{R}\left(T\left(M^{\prime}\right):_{T(M)} I^{n}\right), n \in N
$$

and

$$
\operatorname{Att}_{R}\left(T\left(M^{\prime}\right):_{T(M)} I^{n} / T\left(M^{\prime \prime}\right):_{T(M)} I^{n}\right), n \in N,
$$

are ultimately constant.

Proof. By [7] (1.9), there exists a ring homomorphism $\phi: R \rightarrow S$ such that $S$ is a commutative Noetherian ring and $M$ has an $S$-module structure which is compatible with the $R$-module structure. Also by Lemma 2.5, $F \otimes_{R} S$ is projective relative to $M$ as an $S$-module. Now Let $U(-)=H_{o m}\left(F \otimes_{R} S,-\right)$. Then

$$
T\left(M^{\prime}\right):_{T(M)} I^{n} \cong U\left(M^{\prime}\right):_{U(M)}(I S)^{n}
$$

and

$$
\begin{aligned}
& T\left(M^{\prime}\right):_{T(M)} I^{n} / T\left(M^{\prime \prime}\right):_{T(M)} I^{n} \\
& \cong U\left(M^{\prime}\right): U(M) \\
&(I S)^{n} / U\left(M^{\prime \prime}\right):_{U(M)}(I S)^{n} .
\end{aligned}
$$

Hence by [5] (4.1), we can assume that $R$ is a commutative Noetherian ring. Now by Theorem 3.3,

$$
\begin{aligned}
& \operatorname{Att}_{R}\left(T\left(M^{\prime}\right):_{T(M)} I^{n}\right) \\
= & \left\{P \in \operatorname{Att}_{R}\left(M^{\prime}: M I^{n}\right): P \subseteq Q \text { for some } Q \in \operatorname{Coass}_{R}(F)\right\}
\end{aligned}
$$

and

$$
\begin{aligned}
& \operatorname{Att}_{R}\left(T\left(M^{\prime}\right):_{T(M)} I^{n} / T\left(M^{\prime \prime}\right):_{T(M)} I^{n}\right) \\
= & \left\{P \in \operatorname{Att}_{R}\left(M^{\prime}:_{M} I^{n} / M^{\prime \prime}:_{M} I^{n}\right): P \subseteq Q \text { for some } Q \in \operatorname{Coass}_{R}(F)\right\} .
\end{aligned}
$$

Hence the result follows from $[8,5.4$ and 5.5]. 
Corollary 3.7. Let $I, F, M$, and $T$ be as in 3.6. Then the sequences of sets $A t t_{R}\left(0:_{T(M)} I^{n}\right)$ and $A t t_{R}\left(0:_{T(M)} I^{n} / 0:_{T(M)} I^{n-1}\right)$, $n \in N$ are ultimately constant. Further if $C$ and $D$ denote respectively their ultimate constant values, then

$$
C-D \subseteq \operatorname{Att}_{R}(T(M)) \text {. }
$$

Proof. We can apply the same technique used in proof of $[3,3.6]$. So we omit the proof.

ExAMPLE 3.8. (i) Let $F$ and $M$ be respectively a flat and an Artinian $A$-modules. Then by Remark 3.4, $\operatorname{Hom}_{A}(F, M)$ has a secondary representation and

$$
\begin{aligned}
& \operatorname{Att}_{A}\left(\operatorname{Hom}_{A}(F, M)\right) \\
= & \left\{P \in \operatorname{Att}_{A}(M): P \subseteq Q \text { for some } Q \in \operatorname{Coass}_{A}(F)\right\} .
\end{aligned}
$$

(ii) Suppose $M$ is a non-zero divisible abelian group which is not a flat $Z$ module and let $p$ be a prime number (here $Z$ denotes the ring of integers). Then by [1, page 190, Ex. 10], and Remark $2.1, M$ is projective relative to the Artinian $Z$-module $Z / p^{n} Z$. Hence $\operatorname{Hom}_{Z}\left(M, Z / p^{n} Z\right)$ has a secondary representation and its attached primes can be specified by 3.3 .

\section{References}

[1] Frank W. Anderson and Kent R. Fuller, Categories of Modules (second edition), Springer-Verlag, New York, 1992.

[2] N. Bourbaki, Commutative Algebra, Addison-Wesley, Read. Mass. 1972.

[3] K. Divaani-Aazar and M. Tousi, Asymptotic attached primes related to flat modules, Algebra Colloq. 6 (1999), no. 2, 193-204.

[4] I. G. Macdonald, Secondary representation of modules over a commutative ring, Sympos. Math. XI (1973), 23-43.

[5] L. Melkerson, On asymptatic stability for sets of prime ideals connected with powers of an ideal, Math. Proc. Cambridge Philos. Soc. 107 (1990), 267-271.

[6] Cohomological properties of modules with secondary representation, Math. Scand. 77 (1995), 197-208.

[7] I. Nishitani, Associated prime ideals of the dual of an Artinian module relative to an injective module, Comm. Algebra 22 (1994), 2651-2667.

[8] D. E. Rush, Asymptotic primes and integral closure in modules, Quart. J. Math. Oxford Ser. 43 (1992), no. 2, 477-499.

[9] S. Yassemi, Coassociated primes, Comm. Algebra 23 (1995), 1473-1498.

Department of Mathematics, Faculty of Science, Guilan University, P. O. BOX 1914, RASHT, IRAN

E-mail: ansari@guilan.ac.ir 